\title{
Research on the Existing Problems and Countermeasures of the Cooperative Medical Insurance System for Urban and Rural Residents: A Case Study of a Certain District, Chongqing
}

\author{
Gu Jijian ${ }^{1}$ Gong Jianqiu ${ }^{2}$
}

Chongqing Vocational College of Transportation, District of Jiangjin, Chongqing, 402247

\begin{abstract}
It has been 7 years since the pilot implementation of the cooperative medical insurance system for urban and rural residents began in 2007, and great results have been achieved since implementation, but there are still many problems. This article explores the case of XX District, Chongqing, one of the first pilot cities for cooperative medical insurance for urban and rural residents in the country and analyzes the basic operation and implementation effects of the cooperative medical insurance system for urban and rural residents in XX District, and actually investigates the satisfaction of residents in XX District with the cooperative medical insurance system for urban and rural residents to find out the main problems in the implementation of the medical insurance system and the related countermeasures to improve the cooperative medical insurance system for urban and rural residents.
\end{abstract}

Keywords: urban and rural residents' cooperative medical insurance system; XX District, Chongqing, problems; countermeasures

\section{Introduction}

Jiangbei District, Jiulongpo District, Nan'an District, XX District, and Nanchuan District of Chongqing City, as the first batch of pilot cities for urban and rural residents' cooperative medical insurance in China, launched pilot urban and rural residents' cooperative medical insurance in October 2007, and other districts gradually started in 2008 and 2009. The system covered the whole city and achieved city-level coordination in 2012.

XX District of Chongqing City is the first batch of pilot cities for urban and rural residents' cooperative medical insurance. The author has lived here since childhood and has more contacts with urban and rural residents' cooperative medical insurance system.

Judging from the current status of the implementation of the cooperative medical insurance system for urban and rural residents in $\mathrm{XX}$ District, the various management systems and implementation methods are not yet complete, and many problems have arisen during the actual operation. What are the reasons for these problems and how can we solve them have become issues worthy of research and discussion?
2. Analysis on the operation status of cooperative medical insurance for urban and rural residents in $\mathrm{XX}$ District

\subsection{Analysis of coverage}

According to the Chongqing Urban and Rural Residents' Cooperative Medical Insurance Policy, all the people beyond the urban employee medical insurance coverage are covered by the Urban and Rural Residents' Cooperative Medical Insurance, and in terms of the system design all population has been fully covered ${ }^{[1]}$.

The actual participation rate has also increased year by year from $60.43 \%$ at the beginning of the establishment of the system. In 2013, the number of people participating in the cooperative medical insurance for urban and rural residents in XX District reached 949,921, of which 195,872 were urban residents, 754,049 were rural residents, and 919,288 were covered by Category one insurance, 30,633 people insured by Cat. 2, and the number of college students insured reached 12100 . Overall, the coverage rate has reached $95.05 \%$, and actually full coverage has been achieved (according to international practice, if the coverage rate reaches $90 \%$ and above, the actual coverage rate can be described as full coverage). 


\subsection{Analysis of funding standards}

The funding standard for urban and rural residents' cooperative medical insurance in Chongqing has been improved year by year from 50 yuan/person in 2007 (financial subsidy of 40 yuan/person/year, and individual payment of 10 yuan/person/year). In 2013, Cat. 1 funding of Chongqing urban and rural residents' cooperative medical insurance reaches 340 yuan/person/year (financial subsidy of 280 yuan/person/year, personal payment of 60 yuan/person/year), while Cat. 2 reaches 430 yuan/person/year (financial subsidy of 280 yuan/person/year and personal payment of 150 yuan/person/year).

Of the financial subsidies of 280 yuan/person in 2013, the central financial subsidy was 188 yuan/person, the Chongqing municipal financial subsidy was 69 yuan/person, and the district financial subsidy was 23 yuan/person.

The funding standards and financial subsidy standards have been increasing year by year, ensuring the sustainable development of the cooperative medical insurance system for urban and rural residents ${ }^{[2]}$, and also effectively protecting the basic medical needs of the insured.

\subsection{Medical treatment analysis}

\subsubsection{General outpatient fees}

The quota reimbursement standard for general outpatient expenses has been increased year by year from 20 yuan/person/year in 2007 to 60 yuan/person/year in 2013, and the quota unused or lest can be reserved for the next year.

\subsubsection{Hospitalization payment line}

Since the establishment of the urban and rural residents cooperative medical insurance system to the present, the initial sum paid by the patient himself in the Grade I and Grade II hospitals has still remains up to now to be 100 yuan/time and 300 yuan/time. For Grade III hospital, the initial sum was reduced from $1000 \mathrm{RMB} /$ time, down to 800 yuan/time in 2010, and also maintained at 800 yuan/time in 2013 .

\subsubsection{Maximum ceiling for hospitalization subsidy}

With the year-on-year increase in funding standards and financial subsidy standards, the ceiling for the Cat. 1 hospitalization subsidy has increased from 30,000 yuan/person in 2007 to 80,000 yuan/person, and for the Cat. 2, it has increased from 70,000 yuan/person/year in 2007 to the current 120,000 yuan/person/year, which effectively ensures the medical treatment of patients with serious and severe illness.

\subsubsection{Proportion of hospital reimbursement}

With the year-on-year increase in funding standards and financial subsidies, the proportion of reimbursement for urban and rural residents' cooperative medical insurance in Grade I hospitals was 70\% in 2007, 50\% in Grade II hospitals, and $30 \%$ in Grade III hospitals ${ }^{[3]}$. Reimbursement rate in the Grade I hospitals has been increased to $80 \%$, with that for the Grade II hospitals increased to $60 \%$, and that for the Grade III increased to $40 \%$ (for the Cat. 2 policy risen additionally $5 \%$ on the basis of the Cat. 2), and has remained unchanged to this day.

\subsubsection{Special disease clinic}

The category number of special diseases has increased from 13 in 2007 to 24 now, which has almost doubled. The reimbursement limit for special diseases has increased from the original 500 yuan/person/year to the current 1,000 yuan/person with the category number of diseases increasing by 11 and the limit increasing by 500 yuan.

\subsubsection{Actual hospital reimbursement ratio}

Since the establishment of the cooperative medical insurance system for urban and rural residents in XX District, especially since the coordination on municipal level of Chongqing, with the improvement of various policies, especially the three major directories, the actual reimbursement ratio for urban and rural residents' cooperative medical insurance has increased year by year ${ }^{[4]}$. The level of insurance coverage has been significantly improved from the actual reimbursement ratio of $31.20 \%$ in 2007 to $60.30 \%$ in 2013.

\subsection{Analysis of settlement methods}

XX District's urban and rural residents' cooperative medical insurance settlement method has mainly gone through three stages from the establishment of the system to the present.

The first stage: from 2007 to 2009 , it was mainly settled by project, which was suspected to induce consumers.

The second stage: from 2010 to 2013, the settlement was mainly based on on-site medical expenses, supplemented by indicators such as the proportion of drugs and the average number of days in hospital, which played a positive role in controlling the rapid growth of medical expenses ${ }^{[5]}$. 
The third stage: from 2013 to the present, the total pre-settlement has been implemented. It is understood that through the one-year operation in 2013, the overall situation is still relatively stable, but the actual work of XX District needs to be further improved by collecting related indicators.

\subsection{Analysis of Fund Income and Expenditure}

\subsubsection{Fund income}

In 2013, the income of urban and rural residents' cooperative medical insurance fund of XX District was 32.284 million yuan, of which personal contributions were 56.8393 million yuan, central financial subsidy being 17.841 million yuan, Chongqing municipal financial subsidy 65.81 million yuan, financial subsidy at equal administrative level 21.5654 million yuan, and family planning subsidy 551340 yuan, the civil affairs subsidy 24,590 yuan, and the interest income 3,344,644 yuan.

\subsubsection{Fund expenditure (compensation)}

In 2013, XX District's urban and rural residents' cooperative medical insurance provided 496,000 outpatient visits with a fund expenditure of 39.02 million yuan, and 278,000 special disease outpatient visits with a fund expenditure of 7.96 million yuan; 108,000 person-time outpatient hospitalizations, 19, 387, 700 yuan fund expenditure and actual hospitalization compensation ratio reaching $60 \%$.

Based on an analysis of the current status of cooperative medical insurance for urban and rural residents in $\mathrm{XX}$ District, the author believes that the overall cooperative medical insurance for urban and rural residents in XX District is operating smoothly, in that the urban and rural residents' cooperative medical insurance fund has a slight balance, and the medical treatment of insured patients has been effectively guaranteed. ${ }^{[6]}$. It has made a positive contribution to maintaining the social stability of $\mathrm{XX}$ and promoting economic development.

\section{Survey and Analysis of Satisfaction of Urban and Rural Resident Cooperative Medical Insurance Residents in XX District}

\subsection{Purpose of investigation}

The purpose of this survey is to understand the awareness and satisfaction of urban and rural residents' cooperative medical insurance system in XX District from the perspective of participating residents, and to explore its causes, so as to analyze the implementation process of urban and rural residents' cooperative medical insurance system in XX District, in order to propose relevant countermeasures to improve the system.

\subsection{Investigation contents}

The main contents of this survey include: the basic information of the insured residents, the satisfaction of the insured residents with the insurance treatment, the satisfaction of the insured residents with the medical treatment and the convenience of settlement, and the satisfaction of the insured residents with the service provided by the designated medical institutions, the satisfaction of the insured residents with the service of the agencies, the enthusiasm of the insured residents to participate, and the overall satisfaction of the insured residents with the insurance system in 7 aspects.

The basic information of the insured residents includes five indicators: gender, household registration, age, education level, and average monthly income.

The satisfaction degree of the insured residents with respect to the insurance benefits includes six indicators: the individual participation insurance payment fee, the initial sum paid by the patient himself, maximum ceiling for hospitalization subsidy, the reimbursement ratio, the drugs and diagnosis and treatment items in the catalog, and the categories of special diseases.

The satisfaction level of the insured residents with regard to medical treatment and the convenience of settlement includes three indicators: the convenience of medical settlement in the city, the convenience of medical settlement outside the city, and the convenience of referral. The satisfaction of the insured residents with the service of the designated medical institutions includes four indicators: the professional level of doctors, the awareness of service of medical staff, the conditions of wards, and the conditions of medical equipment ${ }^{[7]}$.

The satisfaction of the insured residents with the service level of the handling agencies includes two indicators: the service attitude of the handling staff and the business level of the handling staff.

The participation enthusiasm of the residents participating in the insurance includes three indicators: the residents' awareness of the policy, whether they are covered by illness, and the level of participation.

\subsection{Survey methods}

Due to the wide area of XX District and the limitation of human, material and financial resources, this survey mainly uses interviews and questionnaires. Survey points were only set up in 2 urban neighborhoods with concentrated population and 2 rural towns with concentrated population in XX District.

The survey included 6 communities in Zhangjiapo, Shengli Road, Xuanhua, Changzhou, Yingjing Road, and Huilong Road, and 6 villages, namely Jinmen, Shisong, Xaingqian, Qishan, Xinjiezi and Songjiang. 15-17 residents were 
surveyed in each community or village. A total of 200 questionnaires were distributed and 200 questionnaires were recovered, of which 190 were valid questionnaires, and the recovery rate reached $100 \%$. The rate reached $95 \%$.

\subsection{Survey results and analysis}

\subsubsection{Basic information of residents participating in the survey}

\subsubsection{Household registration and gender}

Among the 190 insured residents surveyed this time, there were 94 urban hukou residents, including 48 males and 46 females; 86 rural hukou residents, including 41 males and 45 females (see Figure 1 for details).

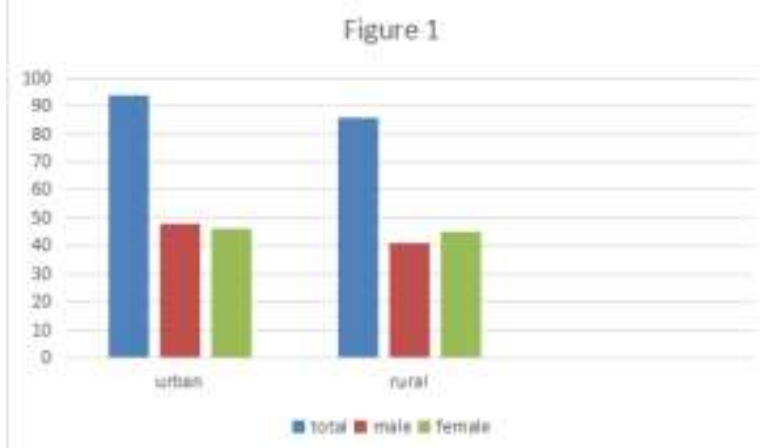

Figure 1 Basic status of household registration and gender of residents participating in the survey

It can be seen from Figure 1 that the proportion of urban household registration and rural household registration among the residents surveyed this time is similar, and the proportion of male and female is also comparable. The questionnaire covers residents with different household registration and gender. The survey result would yield rather fair statistics ${ }^{[8]}$.

\subsubsection{Age profile}

Of the 190 insured residents surveyed, 6 were under the age of 18 , accounting for $3 \%$ of the total number of insured residents under investigation; 37 were in the 18-30 age group, accounting for $19 \%$ of the total number of insured residents under investigation; 64 fall in the 31-45 bracket, accounting for $34 \%$ of the total number of residents participating in the survey; 67 people at the age of 46-60, accounting for $35 \%$ of the total number of residents participating in the survey; 16 people aged 60 or above account for the insurance participants $9 \%$ of total residents; (see Figure 2 for details).





Figure 2 Age of residents participating in the survey

It can be seen from Figure 2 that most of the residents participating in the survey are aged between 18 and 60 Residents in this age group are generally the main labor force in the family and the most important source of family economic income. Their satisfaction with the cooperative medical insurance system for urban and rural residents can be universal and representative.

\subsubsection{Education level}

Among the 190 insured residents surveyed, there are sharp differences in educational level between urban household registration residents and rural household registration residents. (see Figure 3 for details)

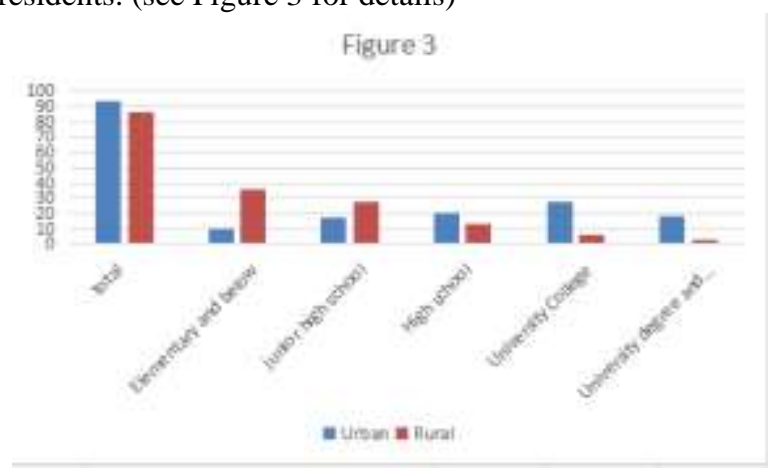

Figure 3 Educational status of residents participating in the survey

\subsubsection{Average monthly income}

Among the 190 insured residents surveyed, 42 had an average monthly income of less than 2, 000 yuan, accounting for $22.1 \%$ of the total number; 81 had an average monthly income of 2000-4000, accounting for $42.6 \%$ of the total number. There were 54 people with average monthly income between 4000 and 6000 , accounting for $28.5 \%$ of the total number of people, and 13 people with an average monthly income above 6000, accounting for $6.8 \%$ of the total number of people (see Figure 4 for details). 


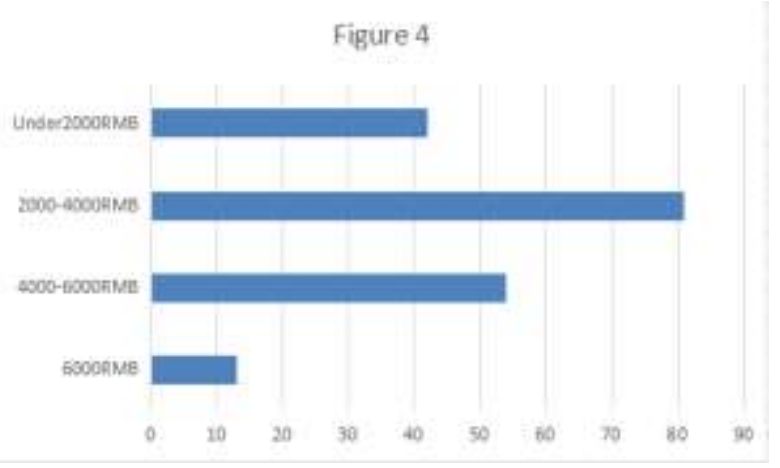

Figure 4 Monthly average income of residents surveyed

It can be seen from Figure 4 that the average monthly income of the residents participating in the survey is mostly 2,000-4,000 yuan; according to the survey results, those with an average monthly income of less than 2,000

Table 1 Satisfaction of the insured residents on the level of insurance benefits

\begin{tabular}{|c|c|c|c|c|c|}
\hline index & Very satisfied & More satisfied & general & Dissatisfied & $\begin{array}{c}\text { Very } \\
\text { dissatisfied }\end{array}$ \\
\hline $\begin{array}{c}\text { Individual insurance } \\
\text { payment fees }\end{array}$ & $16(8.4 \%)$ & $102(53.7 \%)$ & $62(32.6 \%)$ & $10(5.3 \%)$ & $0(0 \%)$ \\
\hline $\begin{array}{c}\text { the initial sum paid by } \\
\text { the patient himself, }\end{array}$ & $14(7.4 \%)$ & $84(44.3 \%)$ & $87(45.7 \%)$ & $5(2.6 \%)$ & $0(0 \%)$ \\
\hline $\begin{array}{c}\text { maximum ceiling for } \\
\text { hospitalization subsidy }\end{array}$ & $16(8.4 \%)$ & $67(35.2 \%)$ & $97(51.1 \%)$ & $10(5.3 \%)$ & $0(0 \%)$ \\
\hline $\begin{array}{c}\text { Reimbursement ratio } \\
\text { Medicines and medical } \\
\text { treatment items }\end{array}$ & $72(6.3 \%)$ & $64(33.7 \%)$ & $97(51.1 \%)$ & $9(4.7 \%)$ & $8(4.2 \%)$ \\
\hline Special disease species & $17(8.9 \%)$ & $121(63.7 \%)$ & $42(22.1 \%)$ & $10(5.3 \%)$ & $0(0 \%)$ \\
\hline
\end{tabular}

It can be seen from Table 1 that in terms of the individual insurance payment fee index, the satisfaction of the insured residents is high, only $5.3 \%$ of the residents are dissatisfied, and none feel absolute dissatisfaction, indicating that for the individuals in XX District, insurance payment fee required are relatively reasonable and can be accepted by most residents.

On the two indicators of the initial sum paid by the patient himself and maximum ceiling for hospitalization subsidy, about $50 \%$ of the insured residents feel satisfied, about $45 \%$ of the insured residents feel average satisfaction satisfaction, and only about $5 \%$ of the insured residents feel dissatisfied and not very dissatisfied. It shows that the cooperative medical insurance for urban and rural residents in XX District is more reasonable in setting the initial sum paid by the patient himself and maximum ceiling for hospitalization subsidy, but it feels that there are also more residents feeling average satisfaction, indicating that both needs to be adjusted.

In the reimbursement ratio indicator, $9 \%$ of the insured residents show a high degree of dissatisfaction, indicating yuan are mainly rural household registration residents, and those above 6,000 yuan are mostly urban household registration residents.

\subsubsection{Satisfaction analysis of insurance residents' insurance treatment}

The satisfaction of the insured residents with the insurance benefits is mainly examined by the six indicators of individual insurance payment fees, the initial sum paid by the patient himself, maximum ceiling for hospitalization subsidy, reimbursement ratios, drugs and diagnosis and treatment items in the catalog, and categories of special diseases (see Table 1 for details). that the reimbursement ratio needs to be increased.

Regarding the medicines and diagnosis and treatment items in the catalog, $15.3 \%$ of the insured residents felt dissatisfied. The degree of dissatisfaction was the highest among the six indicators of insurance benefits, indicating that the medicines and diagnosis and treatment items in the catalog of XX District's urban and rural residents' cooperative medical insurance is not comprehensive enough to meet the medical needs of urban and rural residents.

In terms of the categories of special diseases, $72.6 \%$ of the insured residents were satisfied, and $8.9 \%$ were very satisfied, which is the highest among the six indicators of insurance benefits, indicating the category of special diseases in urban and rural residents' cooperative medical insurance in XX District is reasonable and complete.

In general, the level of cooperative medical insurance coverage for urban and rural residents in XX District is relatively reasonable, which basically meets the needs of insured residents, but there is also room for improvement. 


\subsubsection{Satisfaction analysis of insured residents on medical treatment and convenience of settlement}

The satisfaction of the insured residents with medical treatment and the convenience of settlement is mainly examined by studying the three indicators of convenience of settlement of medical treatment in the city, convenience of medical treatment outside the city, and convenience of referral. (see Table 2 for details).

Table 2 Satisfaction of insured residents on medical treatment and convenience of settlement

\begin{tabular}{|c|c|c|c|c|c|}
\hline index & $\begin{array}{c}\text { Very } \\
\text { satisfied }\end{array}$ & $\begin{array}{c}\text { More } \\
\text { satisfied }\end{array}$ & general & Dissatisfied & $\begin{array}{c}\text { Very } \\
\text { dissatisfied }\end{array}$ \\
\hline $\begin{array}{c}\text { Convenience of city } \\
\text { medical settlement }\end{array}$ & $20(10.6 \%)$ & $121(63.7 \%)$ & $44(23.1 \%)$ & $5(2.6 \%)$ & $0(0 \%)$ \\
\hline $\begin{array}{c}\text { Convenience of medical } \\
\text { settlement outside the } \\
\text { city }\end{array}$ & $9(4.7 \%)$ & $64(33.7 \%)$ & $92(48.5 \%)$ & $17(8.9 \%)$ & $8(4.2 \%)$ \\
\hline Convenience of referral & $17(8.9 \%)$ & $126(66.3 \%)$ & $42(22.1 \%)$ & $5(2.6 \%)$ & $0(0 \%)$ \\
\hline
\end{tabular}

In terms of the convenience index for medical treatment and settlement in the city, the satisfaction rate of the insured residents reached $74.3 \%, 32.2 \%$ of the insured residents felt average satisfaction, and only $2.6 \%$ of the insured residents were not satisfied. The settlement of medical treatment in the insurance city is more convenient. This is mainly attributed to the online real-time settlement for medical treatment in Chongqing.

In terms of the convenience index for settlement of medical treatment outside the city, the satisfaction of the residents participating in the insurance is $38.4 \%$. Most of the residents participating in the insurance feel average satisfaction, and $13.1 \%$ of the residents who feel insured feel dissatisfied. It has dropped a lot, mainly because manual settlement is used for medical settlement outside Chongqing, and the settlement cycle is at least 7-15 days, which is more troublesome.

In terms of convenience of referral, residents' satisfaction has reached more than $75 \%$, mainly because the increased referral convenience and flexibility since municipality-level coordination took effect in Chongqing municipal and rural residents' cooperative medical insurance. Free referral can happen at Grade II hospitals and below. Although referral in the Grade III hospital needs to be proved by the handling agency, timely approval can be ensured.

\subsubsection{Satisfaction analysis of insured residents on the service level of designated medical institutions}

The satisfaction of insured residents with the service of designated medical institutions is mainly examined by studying four indicators of doctors' professional and technical levels, service consciousness of medical staff, ward conditions, and medical equipment conditions. In the inspection of these four indicators ${ }^{[9]}$, urban insured residents and rural insureds residents' satisfaction varies widely (see Figure 5 for details)

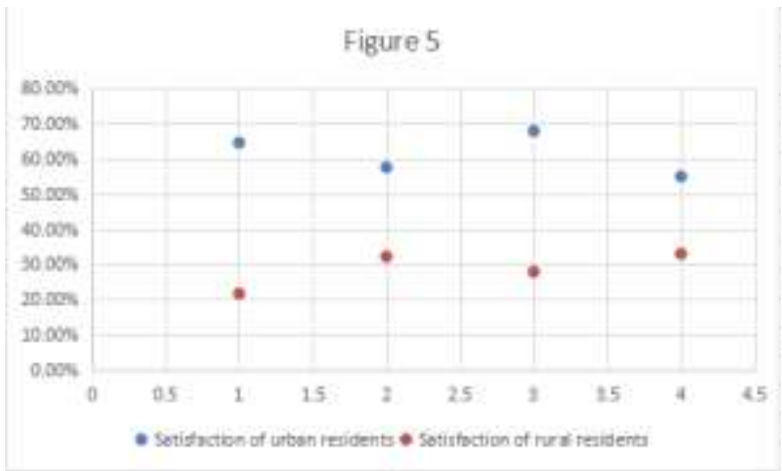

Figure 5 Satisfaction of insured residents with service levels of designated medical institutions

It can be seen from Figure 5 that the satisfaction of urban insured residents on the service level of designated medical institutions is generally higher than that of rural insured residents. This is due to regional gaps in the professional and technical level of medical staff, service consciousness, and facilities of medical institutions in XX District's urban and rural designated medical institutions.

\subsubsection{Satisfaction analysis of insured residents on the service level of the agency}

The satisfaction of the insured residents with the service level of the handling agency is mainly examined by studying the two indicators of the service attitude of the handling personnel and the business level of the handling personnel (see Table 3 for details). 
Table 3 Satisfaction of insured residents to the service level of the agency

\begin{tabular}{|c|c|c|c|c|c|}
\hline index & $\begin{array}{c}\text { Very } \\
\text { satisfied } \\
\text { satisfied }\end{array}$ & $\begin{array}{c}\text { More } \\
\text { general }\end{array}$ & Dissatisfied & $\begin{array}{c}\text { Very } \\
\text { dissatisfie } \\
\mathrm{d}\end{array}$ \\
\hline $\begin{array}{c}\text { Manager's service } \\
\text { attitude }\end{array}$ & $11(5.8 \%)$ & $54(28.4 \%)$ & $101(53.2 \%)$ & $19(10 \%)$ & $5(2.6 \%)$ \\
\hline $\begin{array}{c}\text { Manager's business } \\
\text { level }\end{array}$ & $13(6.8 \%)$ & $66(34.8 \%)$ & $96(50.6 \%)$ & $15(7.8 \%)$ & $0(0 \%)$ \\
\hline
\end{tabular}

It can be seen from Table 3 that the insured residents are less satisfied with the service level of the handling agency, especially with the service attitude. Only $34.2 \%$ of the insured residents are satisfied, and there are those who are very dissatisfied. The service quality of the agency still needs to be continuously improved.

\subsubsection{Analysis of enthusiasm of participating residents}

The enthusiasm of the insured residents for insurance coverage is mainly examined by studying the three indicators of the insured residents' awareness of the policy, whether they are insured with illness, and the level of insurance coverage.

On the indicator of the degree of awareness of the policy-insured residents, 23 insured residents understand very well, 87 residents fairly understand, 62 residents have little understanding, and 18 residents only participate with no understanding at all. It can be seen that the residents show little awareness of this policy.

In terms of insurance with disease, the number of residents reached 117. The insured residents are not actively participating in the insurance. Most residents were passively insured.

In terms of insurance level indicators, 132 insured residents chose Cat. 1 insurance, and only 67 insured residents chose Cat. 2 insurance.

Generally speaking, the participation enthusiasm of the insured residents is very low, especially the rural insured residents.

\subsubsection{Analysis of the overall satisfaction of the insured residents with the insurance system}

In terms of the overall satisfaction of the insured residents with the insurance system, 12 insured residents chose to be very satisfied, and thought that they had benefited a lot since insured; 71 insured residents chose to be more satisfied, and believed that actual problems can be solved since the coverage. 103 insured residents chose to be generally satisfied and thought that it did not have much impact on their life since the coverage; 4 residents chose to be dissatisfied and thought that participating in the insurance was useless
From this point of view, the implementation of the cooperative medical insurance system for urban and rural residents in XX District has played a great role, but it still needs to be continuously improved.

\section{Analysis of the existing problems and countermeasures of the cooperative medical insurance system for urban and rural residents in XX District}

\subsection{Problems in the implementation of the cooperative medical insurance system for urban and rural residents in $X X$ District}

\subsubsection{The level of insurance needs to be further improved}

\subsubsection{The reimbursement ratio needs to be adjusted and improved}

Judging from the satisfaction survey results of the cooperative medical insurance reimbursement ratio of urban and rural residents in XX District, the satisfaction rate is not high with only $40 \%$ of the insured residents feeling satisfied with the reimbursement ratio.

The actual reimbursement ratio of urban and rural residents' cooperative medical insurance system in XX District in 2013 was $51 \%$, of which the actual reimbursement ratio of Grade I medical institutions was $73 \%$, Grade II 53\%, and Grade III $26 \%$.

At present, urban and rural residents' cooperative medical insurance policy stipulates that inpatient reimbursement must differentiate between hospital grades, resulting in different benefit for inpatients according to different standard of proportion of reimbursement and the initial sum paid by the patient himself. The purpose of this regulation is to discourage patients with minor illnesses to go large hospitals ${ }^{[10]}$. The reason behind that is, on the one side, to makes full use of medical resources, relieve the pressure on large hospitals, and promotes the development of small and medium-sized hospitals, especially township hospitals and village health clinics; on the other hand, to 
reduce medical expenses and control medical insurance fund expenditures. But at the same time, a problem arises: those who should have visited and stayed in higher-level hospitals are already seriously ill, but the total medical expenses are relatively high with the actual reimbursement ratio being lower, thus increasing the financial burden. It does not solve the problem of expensive medical treatment, and it does not reflect the essence of the insurance policy: The more serious the illness is, the more important role medical insurance plays. This is also an important factor that contributes to the residents' satisfaction with the reimbursement ratio of urban and rural residents' cooperative medical insurance.

Therefore, the reimbursement ratio of urban and rural residents' cooperative medical insurance needs to be adjusted and increased. At the same time, the actual reimbursement work needs to be strengthened.

\subsubsection{Drugs and diagnosis and treatment items in the catalogue need to be increased}

Since the coordination at the municipal level, Chongqing Municipal and Rural Residents' Cooperative Medical Insurance, the drug catalogue and diagnosis and treatment project catalogues have been integrated with the urban employee medical insurance. It should be said that the medicines and diagnosis and treatment items in the catalogue have been expanded compared to before. However, the drug catalogue still comes in the version of 2009. It has not been adjusted for nearly 5 years; the list of diagnosis and treatment items was developed in 2004. At present, some diagnosis and treatment measures effective and commonly used, such as gamma knives, are beyond the reimbursement scope, resulting in heavy financial burden on insurance participants.

At the same time, as is known by the author, in order to obtain the maximum benefits, some medical institutions in XX District often use some drugs and diagnosis and treatment items outside the catalogue, which increased the burdens of the insured.

\subsubsection{Convenience of medical insurance and settlement needs to be improved}

Chongqing's urban-rural residents' cooperative medical insurance has been coordinated at the municipal level and the city has realized timely coordinated networking, making medical settlement very convenient. However, only Hainan, Zunyi, and Guang'an have achieved cross-provincial online settlement for medical treatment outside the city. The vast majority of provinces and cities do not have access to online settlement, and further cross-provincial networked settlements or even national networked settlements need to be further expanded.

\subsubsection{The quality of medical services and medical insurance agencies in designated medical institutions need to be improved}

Although in $\mathrm{XX}$ the number of designated medical institutions and designated retail pharmacies in the network system of urban and rural residents' cooperative medical insurance has reached 750 (including 350 village clinics), the service quality of designated medical institutions has been uneven. There is overall low level of satisfaction based on results of satisfaction surveys on the service levels of designated medical institutions by participating residents. urban residents and rural residents have greater differences in satisfaction with the service level of designated medical institutions. The satisfaction of urban residents is generally higher than that of rural residents, which indicates a large gap between urban and rural designated medical care service. The service level of designated medical institutions in rural areas needs to be improved urgently. At the same time, the service level of designated medical institutions in urban areas must also be continuously improved ${ }^{[11]}$.

Regarding the reasons for the low level of satisfaction of residents with the service level of medical insurance agencies, the author believes that the staffing of the agency is seriously insufficient, which affects the quality of services as the main reason.

In accordance with international practice, there should be 1-2 handling personnel for every 10,000 people, then 949,000 insured personnel in XX District should be equipped with 95-190 handling personnel. There are 37 staff members in XX District Medical Insurance Center. Agency staffing is severely inadequate.

Therefore, the service quality of both the designated hospital and the agency needs to be improved.

\subsubsection{Some residents don't have a strong sense of enrollment}

Residents' awareness of and motivation for insurance coverage needs to be raised. Most of the residents with illness are participating in the insurance scheme. Meanwhile, most of them choose Cat. 1 insurance, which is common among rural residents.

The author believes that the residents' lack of insurance participation is mainly caused by the following reasons: First, some residents have insufficient knowledge of the major diseases that may occur. They believe that major diseases will not fall on their own families ${ }^{[12]}$. The family has tolerance for minor problems and has not formed a group awareness of active participation in insurance.

Second, the relevant departments have not engaged in adequate and proper publicity on the cooperative medical insurance system for urban and rural residents, resulting in insufficient awareness of the cooperative medical insurance system for urban and county residents (especially rural residents), and affecting residents' willingness to participate in insurance. 
Third, the average education level of rural residents is low, and the risk awareness is not strong. In addition, the average income level of rural residents is low, resulting in the majority of rural residents choosing low-level insurance coverage.

Fourth, the service level of designated medical institutions in rural areas is low, which affects the enthusiasm of rural residents for insurance.

\subsubsection{The presence of a large number of vacant people and the presence of unreturned personnel who have been away all year round have affected insurance participation and fundraising}

There is a large number of empty people, which affects insurance and fund-raising work. In the 1990s of XX District, there were many people who bought urban hukou from the agricultural population, and there are still some people who are not in the household. According to statistics, among more than 1,300 households in the district, more than 28,500 people are vacant. These people remain unknown and their location unclear. Neither the residents' committee nor the resident group cadres can contact them, and their effective participation in insurance cannot be organized, which will affect the participation and fundraising.

The presence of unreturned personnel for many years has affected insurance and fund-raising work. According to statistics, more than 300,000 people in XX District go out to work every year. Due to the large social mobility, the address, working units and telephone numbers change often, making it impossible for the community to contact them and making them unable to be effectively covered by insurance, which affected the participation and fundraising work.

\subsubsection{It is difficult to collect medical insurance funds, which leads to the phenomenon of collecting ordinary outpatient fees by deceiving.}

The main cause of this problem is that medical insurance departments at all levels are assigned sales quotas every year. However, in the process of excluding empty population, dead registered population, long-term out-of-town employees, migrant workers, and urban employees and those participating in urban employee medical insurance and flexible employment medical insurance, the village cadres, in order to complete the quotas, must pay in advance for those excluded to complete the tasks. However, the payment in advance by village cadres cannot be recovered, and they have worked with the village clinic to find a way to collect outpatient fees.

\subsection{Thoughts on Improving the Cooperative Medical Insurance System for Urban and Rural Residents}

\subsubsection{Further increasing the level of insurance}

(1) The competent government department should seriously study and further expand the drug list and the diagnosis and treatment measure list. The two lists should be adjusted in a timely manner to include the drugs and diagnosis and treatment measures effectively and commonly used into the scope of reimbursement.

(2) Reduce or cancel funding standards, cancel outpatient charges for they can be afforded by ordinary families can afford it, and there will be no poverty due to illness and return to poverty due to illness.

(3) With the gradual increase of the three-level financial subsidy standards, the proportion of hospital reimbursement for hospitals at all levels should be increased in a timely manner. Only inpatient treatment will incur large costs. If patients with serious illnesses do not receive funding from the medical insurance benefit policy, poverty will naturally set in due to illness and the patients will return to poverty due to illness. Only by increasing the proportion of inpatient reimbursement can the insurance play a medical security role.

(4) Further strengthen the supervision of medical institutions. Clinicians should try to use the medicines and diagnosis and treatment measures in the catalog as much as possible, and to use Class A medicines and diagnosis and treatment measures in the catalog as much as possible to increase the reimbursement ratio.

\subsubsection{Simplify the procedures and facilitate the participants}

Simplify the various procedures of urban and rural residents' cooperative medical insurance, from application, payment, hospitalization, reimbursement to transfer, thus strengthening the flexibility of the working mechanism.

(1) Improve financing methods by withholding and remitting through economic and financial departments. Many people-friendly policies now implement one-card access. By integrating the basic information of family members in one card, one can realize withholding and remitting by economic and financial departments if they wish, ensuring convenience of payment anywhere and avoid neglect. In this way the insurance system can operate effectively.

(2) What should be studied at the national level is the transfer continuity of residents' medical insurance account across provinces and the implementation of national networked timely settlement.

(3) Hospitals with centralized diagnosis of special diseases should be added. It is known that there are only three hospitals qualified to diagnose special diseases, $\mathrm{XX}$ Hospital, XX District Disease Control Center (only for 
treating tuberculosis and AIDS), and $\mathrm{XX}$ District Psychiatric Hospital (only for treating mental illness). Eligible secondary hospitals should be included in the medical institutions with centralized diagnosis of special diseases.

\subsubsection{Further increasing investment in township hospitals and community health services}

Investment in township hospitals should further be increased. We should improve medical facilities and equipment, training and transportation of medical staff in township hospitals, and improve the medical skills of medical staff, so that those insured can stay in township medical institutions and community health after they become ill. In doing that, people with minor illnesses do not need to leave the village, and generally do not need to leave the town, which reduces the medical burden of the insured and benefit them more and thereby changing the current situation of difficulties and high expense in medical care.

It is understood that the medical needs of insured residents can be basically met by the equipment in township hospitals and community health services in XX District, and the professional and technical level of medical staff. However, most of the branches of those hospitals and centers cannot meet the medical needs of ordinary people, for their old and backward equipment and a serious shortage of professional and technical personnel ${ }^{[13]}$. For example, the Yongan Branch of Ji' an Town Health Center does not have a piece of medical equipment and the shortage of personnel affects the medical needs of 13,000 people.

\subsubsection{Strengthening the supervision and management of medical services at designated medical institutions and improve the quality of medical services}

It is understood that some medical treatments in $\mathrm{XX}$ District include violations such as hanging bed hospitalization, decommissioning hospitalization, empty bed hospitalization, minor disease hospitalization, illegal inspection and medication, which not only affects the interests of residents but also threatens the safe operation of the medical insurance fund ${ }^{[14]}$. The supervision and management of designated medical institutions should be further strengthened to improve the quality of hospital services and ensure the safe operation of funds.

(1) The administrative department of health should further improve ethics quality in health care, so that the majority of medical staff can establish good medical ethics to ensure the safe operation of the medical insurance fund.

(2) The social and human resources department should further improve the rules and regulations of the service agreements and medical insurance of designated medical institutions, establish an entry and exit mechanism for designated medical institutions, and implement "the survival of the fittest" policy.

(3) The human, social and health departments, and other departments should strengthen timely monitoring of designated medical institutions on the Internet and take measures such as clear investigations and unannounced visits, special inspections and other means to strengthen on-site supervision and inspection of designated medical institutions, and discover violations together. Serious punishment should be carried out once foul play is detected.

\subsubsection{Government departments should further strengthen the capacity building of handling agencies}

(1) The government establishment department should increase the staffing of the medical insurance center properly, based on the continuous increase in the number of participants, especially the medical, legal, and network professionals to improve its service and management level. (2) The financial department should increase investment in handling agencies. Financing of residents' medical insurance is very difficult, yet still a must. Investment should be increased in town (street) social insurance offices and district medical insurance centers to improve the management staff's motivation to ensure the completion of insurance tasks, thereby ensuring the safety of the fund.

(3) The handling agency should further strengthen its own construction, building its public image, strengthen its internal quality, and strive to improve the policy awareness and ability of each handling staff, so as to better provide quality services to the insured.

\subsubsection{Continuously strengthening publicity and raise residents' awareness of insurance participation}

To increase residents' awareness of insurance participation, it is necessary to strengthen residents' awareness of urban and rural residents' cooperative medical insurance system.

Therefore, relevant departments should promote the cooperative medical insurance system for urban and rural residents, so that residents can have more channels to easily understand the system.

The author believes that under the continuous development of today's information-oriented society, it is necessary not only to use traditional methods such as television and newspapers for publicity, but also to resort to online and outdoor field promotion. Publicity columns for the system can be set up in urban areas with concentrated populations. On-site publicity activities and consultation can be set up. At the same time, such columns can be set up on relevant websites, and related policy information can be posted there in a timely manner. Residents can consult and discuss, 
and special personnel can engage in the consultation and collect opinions online.

In this way, residents can not only deepen their basic understanding of the cooperative medical insurance system for urban and rural residents, but also understand the operation of the system in a timely manner. At the same time, they can also provide residents with timely feedback and suggestions.

Only by increasing the residents' awareness of the urban and rural residents' cooperative medical insurance system, and allowing the residents to supervise and advise on the implementation of the system, can the residents' awareness of active participation and the participation rate be increased.

\section{Conclusion}

In short, the development of medical cooperatives still needs a lot of improvement from the perspective of results. In particular, the level of participation, the knowledge of the insured personnel on the policy and what the results will bring, all need to be taken seriously, not sloppy, otherwise Your health rights and interests cannot be fully maintained, and in the end, you will lose the gains.

\author{
About the author \\ Gu Jijian, male, 1980.8, Cheng Wuren of Shandong, \\ professor, postdoctoral and master's tutor of Chongqing \\ Vocational College of Transportation, mainly studying \\ macroeconomic policies, institutional changes, and \\ economic performance \\ Address: No. 555 Xiangfu Avenue, Shuangfu Street, \\ Jiangjin District, Chongqing \\ Postcode: 402247 \\ Contact: 15683022233
}

\section{REFERENCES}

[1] Li Jin. Research on the integration of urban and rural residents' medical insurance system [d]. Capital University of Economics and Business, 2011.

[2] Li Yuan, Huang Zhaohui, Shi Kai, Yu Xuan. An Empirical Study of Cooperative Medical Insurance for Urban and Rural Residents in Chongqing [j]. Journal of PLA Hospital Management, 2011, 01: 49-51.

[3] Yang Xiaoli. Practical exploration of Chongqing's overall urban and rural medical security system [j]. China Health Economics, 2011, 04: 26-28.

[4] Yang Xiaoli. Practical exploration on the integration of urban and rural medical security in Chongqing [j].
China Health Service Management, 2011, 05: 343-344 +355 .

[5] Ma Weizheng, He Ning. Comparative analysis of overall urban and rural medical insurance system model [j]. Theory and Modernization, 2012, 05: 64-69.

[6] Xiang Chunling. Establishing an urban-rural integrated medical security system-A survey of Chongqing's urban-rural integrated medical security system [j]. Journal of the Party School of the Central Committee of the Communist Party of China, 2009, 02: 100-104.

[7] Chen Lin, Zhang Lianguang. A Case Study on the Integrated Medical Care Model for Urban and Rural Residents [j]. Business Times, 2009, 15: 57-58.

[8] Yu Ruijun, Ma Xinli. Preliminary Study on the Design of Urban and Rural Coordinated Medical Security System [j]. Modern Finance and Economics (Journal of Tianjin University of Finance and Economics), 2009, 11: 24-29.

[9] Miao Yanqing, Wang Lusheng. Case study of basic medical security system for urban and rural residents: pilot practice and main findings [j]. China Health Policy Research, 2010, 04: 9-16.

[10] Shen Qin, Yu Zhongwei, Hu Hao. The main methods of urban-rural integrated medical insurance system in Jiaxing [j]. China Health Policy Research, 2010, 04: 32-36.

[11] Liang Ping, Peng Jialing. Comparison of urban and rural medical insurance systems in Chongqing [j]. Cooperative Economy and Science and Technology, 2010, 10: 108-110.

[12] Shen Qin. Exploration and practice of the integration of the new rural cooperative medical service and urban residents' medical insurance in Jiaxing City, Zhejiang Province [j]. Chinese Health Policy Research, 2013, 02: 14-18.

[13] Zhou Zhongchang. Research on Urban and Rural Coordination of Medical Insurance in China [d]. Shandong University of Finance and Economics, 2012.

[14] Li Zhiying. Study on the Linkage of Urban and Rural Basic Medical Insurance System in China [d]. Central China Normal University, 2011. 